\title{
A comparative study of effect of fluoroquinolones on blood glucose levels in rats
}

\author{
Tanya Gupta ${ }^{1}$, Sarita Mulkalwar ${ }^{1 *}$, Vishwanath Kulkarni ${ }^{1}$, \\ Sudhir Jadhav ${ }^{2}$, Abhijeet Tilak ${ }^{1}$, Bhalchandra Rane ${ }^{1}$
}

\begin{abstract}
${ }^{1}$ Department of Pharmacology, ${ }^{2}$ Department of Preventive and Social Medicine, Dr. D. Y. Patil Medical College, Hospital and Research Centre, Pimpri, Pune, India
\end{abstract}

Received: 27 November 2019

Revised: 14 June 2020

Accepted: 17 June 2020

\section{*Correspondence:}

Dr. Sarita Mulkalwar,

Email: sarita.mulkalwar@dpu.edu.in

Copyright: (C) the author(s), publisher and licensee Medip Academy. This is an open-access article distributed under the terms of the Creative Commons Attribution Non-Commercial License, which permits unrestricted non-commercial use, distribution, and reproduction in any medium, provided the original work is properly cited.

\section{ABSTRACT}

Background: Fluoroquinolones (FQ) are frequently prescribed because of their broad-spectrum applicability in treatment of community acquired pneumonia and urinary tract infections. Increased use has raised some concerns regarding side effects like dysglycaemia, tendon rupture and QT interval prolongation. Gatifloxacin was banned in India in 2011 for causing fatal hypoglycemia. This study compares the effect of different fluoroquinolones on blood glucose levels in rats.

Methods: 24 rats were divided into four study groups. Each group was administered one fluoroquinolone namely levofloxacin $9 \mathrm{mg}$, moxifloxacin $7.2 \mathrm{mg}$, ciprofloxacin $18 \mathrm{mg}$ and ofloxacin $14.4 \mathrm{mg}$ respectively for five days. The changes in blood glucose levels were observed for 10 days.

Results: The mean blood glucose levels in all the four groups dropped below the baseline by day five. A statistically significant reduction in mean glucose levels was found in the moxifloxacin, ciprofloxacin and levofloxacin group. Among the rats that were given ofloxacin, the reduction in the mean blood glucose levels was not statistically significant. After stopping the drugs, the blood glucose levels in all the four groups returned near to the baseline within five days.

Conclusions: The use of fluoroquinolones causes hypoglycemia in rats. The blood glucose level reduction associated with moxifloxacin was maximum, whereas ofloxacin appeared to have the minimum effect on blood glucose levels. These effects do not appear to be permanent and the dysglycaemia subsided after the drugs were stopped.

Keywords: Fluoroquinolones, Hypoglycemia, Moxifloxacin, Levofloxacin, Blood glucose, Rats

\section{INTRODUCTION}

It's been nearly half a century since fluoroquinolones have been developed by modifying the fluoro group at position 6 of nalidixic acid to improve the gram-positive bacteria activity of quinolones, an antibiotic category already fairly potent against gram-negative bacteria. The older ones like ciprofloxacin and norfloxacin are primarily used to treat urinary tract infections (UTI) infections whereas the newer ones like moxifloxacin are extremely potent against the bacteria causing respiratory tract infections e.g. S. pneumoniae, $H$. influenzae and $M$. catarrhalis. Although new and improved fluoroquinolones have been fairly regularly introduced into the market, they were not without side effects, some of them being tendon rupture, QT interval prolongation, and dysglycemia. ${ }^{1}$ Trovafloxacin has been withdrawn from the market because it lead to life threatening hepatotoxicity. ${ }^{2}$ Grepafloxacin was withdrawn because of many reports of it being associated with sudden cardiac death. ${ }^{3}$ Perhaps the most significant was the side effects 
of gatifloxacin. In a study conducted on 788 patients, gatifloxacin was associated with a 4.3 times higher odds of hypoglycemia than macrolides and 16.7 times higher odds of hyperglycemia than macrolides. ${ }^{4}$ The drug was eventually withdrawn from the US market in 2006 and from India in 2011.5 Gatifloxacin was not the only fluoroquinolone to cause dysglycemia, there have been several case reports showing the risk of hypoglycemia in patients on levofloxacin and moxifloxacin..$^{6-11}$

Several case reports have indicated the severe hypoglycemic effect of use of fluoroquinolones, but only few studies have shown the comparison of different fluoroquinolones in terms of their effect on blood glucose. It remains unclear that which fluoroquinolone has more effect on blood glucose levels. Also, human studies comparing the dysglycemic effects of different fluoroquinolones were few in number and had multiple confounding factors. Hence, an animal model was chosen to demonstrate and compare the effect of the commonly used fluoroquinolones on fasting blood glucose. Prescribing safe antimicrobial agents with regard to their effects on glucose homeostasis is important, due to the risk of dysglycemic episodes associated with infection or sepsis. The aim of this study was to compare the effect of different fluoroquinolones namely levofloxacin, moxifloxacin, ciprofloxacin and ofloxacin on blood glucose levels in rats.

\section{METHODS}

24 experimentally naive Wistar rats of either sex, weighing 150-200 grams, housed in polypropylene cages were procured from the animal house at Dr. D. Y. Patil Medical College, Hospital and Research Centre, Pimpri, Pune. They were maintained under standard conditions of temperature $25 \pm 5^{\circ} \mathrm{C}$ and relative humidity $55 \pm 10 \%$ along with 12 hours night and day cycle. The rats were fed on a standard pellet diet and had free access to water ad libitum. The study was carried out in accordance with the guidelines given by committee for the purpose of control and supervision of experiments on animals (CPCSEA), New Delhi (India) and was approved by Institutional Animal Ethic Committee (IAEC) of Dr. D.Y. Patil Medical College, Hospital and Research Centre, Pimpri, Pune.

The animals were randomly allocated into four groups with 6 rats per arm. Each group was administered a different study drug orally daily for a period of 5 days. All the study drugs used were in tablet form and were generic in nature, obtained from the pharmacy store at D. Y. Patil Medical College, Hospital and Research Centre, Pimpri.

The fasting blood glucose was measured using accucheck active glucometer on the day 0 (baseline) and then every day till the drugs were delivered (for 5 days). After the drugs were stopped the blood glucose levels were measured again on day 7 and day 10. Blood sample was collected from tail vein.

Statistical analysis was done using SPSS (statistical package for social sciences) version 17. All data represent the mean and SD. The ANOVA was use to find significant difference of blood glucose level between the four groups followed by multiple group comparison with post hoc Tukey's test. The repeated measures of ANOVA were used to find the significant difference of blood glucose levels between different time period in each group followed by pair wise comparison with post hoc Bonferroni's test for each group. P value of less than 0.05 was considered as statistically significant.

Table 1: Dosage of drugs.

\begin{tabular}{|lll|}
\hline Groups & Drug & $\begin{array}{l}\text { Dosage } \\
\text { (mg/day) }\end{array}$ \\
\hline Group I & Levofloxacin (L) & 9 \\
\hline Group II & Moxifloxacin (M) & 7.2 \\
\hline Group III & Ciprofloxacin (C) & 18 \\
\hline Group IV & Ofloxacin $(\mathrm{O})$ & 14.4 \\
\hline
\end{tabular}

\section{Ethical approval}

Institutional animal ethics committee and institutional scientific committee approval received.

\section{RESULTS}

There was no significant difference of blood glucose level at day 0 between the study groups so the groups were comparable. Statistical analysis showed that the mean blood glucose levels in all groups decreased from day 0 to day 7 and reached back to near baseline by day 10. A repeated measures analysis done in all the four groups with a green-house Geisser correction, determined that the mean blood glucose level differed statistically between time points in the levofloxacin, moxifloxacin and ciprofloxacin group. In the group which was administered ofloxacin, the difference in the mean blood glucose level existed but was not found to be statistically significant (p value 0.08) (Table 2). Pairwise comparison with Bonferroni's test was done in the study groups and repeated measures ANOVA was performed for each group individually, and it was observed that the groups which were given levofloxacin, moxifloxacin and ciprofloxacin had a statistically significant difference in blood glucose levels between time periods ( $p$ value of $0.001,0.0001$ and 0.0001 respectively on day 7 ).

The blood glucose lowering effect observed in our study groups was compared by Tukey's test and it was seen that, on day 5 , the reduction in glucose levels with levofloxacin was statistically significant as compared to moxifloxacin and ofloxacin, whereas on day 10 the glucose lowering effect with ciprofloxacin was statistically significant as compared to ofloxacin (Table 3 and 4). 
Table 2: Effect of fluoroquinolones on blood glucose level in study groups.

\begin{tabular}{|lllllllllll|}
\hline & \multicolumn{2}{l}{ Levofloxacin } & \multicolumn{2}{c|}{ Moxifloxacin } & \multicolumn{2}{c|}{ Ciprofloxacin } & Ofloxacin & & & \\
BSL & Mean & SD & Mean & SD & Mean & SD & Mean & SD & & P value \\
\hline Day 0 & 112.83 & 8.18 & 110.83 & 6.52 & 109.50 & 5.82 & 104.67 & 5.61 & 1.66 & 0.21 \\
\hline Day 1 & $96.50^{*}$ & 11.31 & 101.67 & 2.58 & 103.17 & 4.07 & 105.83 & 5.27 & 2.07 & 0.14 \\
\hline Day 3 & 102.83 & 3.31 & 109.83 & 5.60 & 106.00 & 4.94 & 105.17 & 8.86 & 1.40 & 0.27 \\
\hline Day 5 & $94.67^{*}$ & 6.28 & 110.83 & 10.68 & 102.50 & 4.93 & 112.17 & 2.04 & 8.72 & 0.001 \\
\hline Day 7 & $92.67^{*}$ & 9.67 & $87.17^{*}$ & 7.65 & $93.83^{*}$ & 6.55 & 96.67 & 7.74 & 1.49 & 0.25 \\
\hline Day 10 & 101.33 & 2.34 & 103.17 & 2.23 & 99.67 & 1.21 & 104.83 & 4.75 & 3.49 & 0.035 \\
\hline
\end{tabular}

*Significant reduction in the blood glucose levels as compared to the baseline.

Table 3: Comparison of effect on blood glucose level between study groups by Tukey’s test.

\begin{tabular}{|lllllll|} 
& \multicolumn{2}{l}{ Blood glucose level } & & & & \\
& Day 0 & Day 1 & Day 3 & Day 5 & Day 7 & Day 10 \\
Groups & P value & P value & P value & P value & P value & P value \\
\hline L v/s M & 0.95 & 0.55 & 0.22 & $0.003^{*}$ & 0.64 & 0.70 \\
\hline L v/s C & 0.82 & 0.34 & 0.80 & 0.22 & 0.99 & 0.76 \\
\hline L v/s O & 0.17 & 0.11 & 0.91 & $0.001^{*}$ & 0.82 & 0.20 \\
\hline M v/s C & 0.98 & 0.98 & 0.69 & 0.17 & 0.49 & 0.20 \\
\hline M v/s O & 0.39 & 0.71 & 0.55 & 0.99 & 0.20 & 0.76 \\
\hline C v/s O & 0.59 & 0.90 & 0.99 & 0.094 & 0.93 & $0.03^{*}$ \\
\hline
\end{tabular}

*Statistically significant reduction in the blood glucose levels. L: Levofloxacin, M: Moxifloxacin, C: Ciprofloxacin, O: Ofloxacin

Table 4: Pair wise comparison of blood glucose levels in the study groups.

\begin{tabular}{|llll|}
\hline Drugs & Days & Mean difference & P value \\
\hline \multirow{5}{*}{ Levofloxacin } & Day 0 to day 1 & 16.333 & 0.012 \\
\hline & Day 0 to day 3 & 10.000 & 0.442 \\
\hline & Day 0 to day 5 & 18.167 & $0.004^{*}$ \\
\hline & Day 0 to day 7 & 20.167 & $0.001^{*}$ \\
\hline & Day 0 to day 10 & 11.500 & 0.200 \\
\hline \multirow{5}{*}{ Moxifloxacin } & Day 0 to day 1 & 9.167 & 0.327 \\
\hline & Day 0 to day 3 & 1.000 & 1.000 \\
\hline \multirow{5}{*}{ Ciprofloxacin } & Day 0 to day 5 & 0.000 & 1.000 \\
\hline & Day 0 to day 7 & 23.667 & $<0.0001^{*}$ \\
\hline & Day 0 to day 10 & 7.667 & 0.780 \\
\hline \multirow{5}{*}{ Ofloxacin } & Day 0 to day 1 & 6.333 & 0.487 \\
\hline & Day 0 to day 3 & 3.500 & 1.000 \\
\hline & Day 0 to day 5 & 7.000 & 0.286 \\
\hline & Day 0 to day 7 & 15.667 & $<0.0001^{*}$ \\
\hline & Day 0 to day 10 & 9.833 & 0.023 \\
\hline & Day 0 to day 1 & -1.167 & 1.000 \\
\hline & Day 0 to day 3 & -0.500 & 1.000 \\
\hline & Day 0 to day 5 & -7.500 & 0.630 \\
\hline & Day 0 to day 7 & 8.000 & 0.463 \\
\hline & Day 0 to day 10 & -0.167 & 1.000 \\
\hline
\end{tabular}

*Statistically significant reduction in the blood glucose levels.

\section{DISCUSSION}

The present study was conducted with the aim to compare the glucose lowering effect of levofloxacin, moxifloxacin, ciprofloxacin and ofloxacin in rats. While studying the effect of fluoroquinolones on blood glucose level, enoxacin, lomefloxacin, norfloxacin, ofloxacin, levofloxacin and gatifloxacin have been reported to induce hypoglycemia by many authors. ${ }^{12}$ Ofloxacin, gatifloxacin and moxifloxacin have been reported to induce hyperglycemia. ${ }^{13}$ Yasuyoshi Ishiwata, in their study reported that the concentration of serum glucose increased by approximately $140 \mathrm{mg} / \mathrm{dl}$ at 15 minutes after intravenous injection of moxifloxacin at $100 \mathrm{mg} / \mathrm{kg} .{ }^{14}$ 
Yasuyoshi Ishiwata, observed hypoglycaemia in rats in their study after administration of levofloxacin. ${ }^{15}$ Acharya et al reported statistically significant hypoglycaemia in rats in levofloxacin group on $3^{\text {rd }}, 7^{\text {th }}$ and $14^{\text {th }}$ day. Hori et al observed hypoglycemia due to levofloxocin and ciprofloxacin in mice. ${ }^{16,17}$

In our study, it was evident from the results that the mean blood glucose level decreased from day zero to day seven and then again started to increase in all study groups. The glucose lowering effect compared to baseline was statistically significant with levofloxacin, moxifloxacin and ciprofloxacin. There was no significant reduction in blood glucose level with ofloxacin. Comparison of reduction in blood glucose levels between the study groups was done. It was observed that levofloxacin caused statistically significant reduction in blood glucose levels when compared to moxifloxacin and ofloxacin on day five. Ciprofloxacin caused a statistically significant reduction in blood glucose levels when compared with ofloxacin on day 10 . Nora et al in their study revealed that disturbed glucose transporter function may be the cause of the dysglycemic effects of ciprofloxacin. ${ }^{19}$ In 2015, study done by Kabbara et al, 118 patients receiving broad-spectrum fluoroquinolones were followed for six months period. Dysglycemia was more frequently encountered with ciprofloxacin, followed by levofloxacin and moxifloxacin. Hyperglycemia was more common than hypoglycemia in all groups. The highest incidence of hyperglycemia occurred with levofloxacin, followed by ciprofloxacin and moxifloxacin. ${ }^{20}$ Although the hypoglycemia associated with fluoroquinolones was found in many studies the concomitant hypoglycemic drug treatment was a major interacting factor, as majority of the patients were taking insulin and/or other antidiabetic drugs. A systematic review published by Murad et al states that very low quality of evidence substantiates the association between hypoglycaemia and the use of numerous nondiabetic drugs. For gatifloxacin, quality of evidence for drug-induced hypoglycemia was moderate while for the other quinolones (ciprofloxacin, clinafloxacin, levofloxacin, moxifloxacin, and sparfloxacin) the quality of evidence was very low. ${ }^{21}$ The effect of the fluoroquinolones on insulin secretion has been studied by many authors, and the results suggest that these antibiotics cause hypoglycemia by increasing insulin release via blockade of adenosine triphosphate sensitive $\mathrm{K}+$ channels in the $\beta$ cells of the pancreas. ${ }^{18}$

\section{CONCLUSION}

In reference to above mentioned results and discussion it was observed that the fluoroquinolones have blood glucose lowering effects in rats. The glucose lowering effect was more with levofloxacin followed by moxifloxacin and ciprofloxacin respectively. While ofloxacin had limited effect on the blood glucose levels. These effects did not appear to be permanent and the dysglycaemia subsided after the drugs were stopped on day five. From the above observations, it can be concluded that ofloxacin could be a preferred antibiotic in patients with impaired glucose metabolism. Clinicians should also be aware of potential glucose lowering activity of fluoroquinolones specially levofloxacin, moxifloxacin and ciprofloxacin while prescribing in patients with impaired glucose metabolism and on concomitant hypoglycaemic drugs. However, more clinical studies need to be conducted to support the findings.

\section{Funding: No funding sources}

Conflict of interest: None declared

Ethical approval: The study was approved by the Institutional Ethics Committee

\section{REFERENCES}

1. Emmerson AM, Jones AM. The quinolones: decades of development and use. J Antimicrobial Chemotherapy. 2003;51(1):13-20.

2. Chen HJ, Bloch KJ, Maclean JA. Acute eosinophilic hepatitis from trovafloxacin. New England J Med. 2000;342:359-60.

3. Ball P. Quinolone-induced QT interval prolongation: a not-so-unexpected class effect. J Antimicrobial Chemotherapy. 2000;45:557-9.

4. Wyllie PLY, Juurlink DN, Kopp A, Shah BR, Stukel TA, Stumpo C, et al. Outpatient gatifloxacin therapy and dysglycemia in older adults. New England $\mathbf{J}$ Med. 2006;354(13):1352-61.

5. Ahmad A, Patel I, Sanyal S, Balkrishnan R, Mohanta GP. A study on drug safety monitoring program in India. Indian J Pharmaceutical Sciences. 2014;76(5):379.

6. Lawrence KR, Adra M, Keir C. Hypoglycemiainduced anoxic brain injury possibly associated with levofloxacin. J Infect. 2006;52:177-80.

7. Singh M, Jacob JJ, Kapoor R, Abraham J. Fatal hypoglycemia with levofloxacin use in an elderly patient in the post-operative period. Langenbecks Arch Surg. 2008;393:235-8.

8. Friedrich LV, Dougherty R. Fatal hypoglycemia associated with levofloxacin. Pharmacotherapy. 2004;24:1807-12.

9. Gibert AE, Porta FS. Hypoglycemia and levofloxacin: a case report. Clin Infect Dis. 2008;46:1126-7.

10. Kapoor R, Blum D, Batra A, Varma N, Lakshmi K, Basak P, et al. Life-Threatening Hypoglycemia with Moxifloxacin in a Dialysis Patient. J Clin Pharmacol. 2012;52(2):269-71.

11. Mandavia R, Virpariya MM, Patel TK, Tripathi CB. Moxifloxacin-induced hypoglycemia in a nondiabetic patient. Current Drug Safety. 2012;7(2):183-5.

12. Kobayashi N, Tsuhako Y, Matsuyama R, Fujinaga M, Nakamoto M. Hypoglycemic attacks in three patients with non-diabetic chronic renal failure on hemodialysis, with discussion of a patient who 
showed enoxacin-induced hypoglycemia. J Japan Soc Dial Ther. 1991;24:951-6.

13. Tack KJ, Smith JA. The safety profile of ofloxacin. Am J Med. 1989;87(6C):78-81.

14. Ishiwata $Y$, Takahashi $Y$, Nagata M, Yasuhara M. Effects of Moxifloxacin on Serum Glucose Concentrations in Rats. Biol Pharm Bull. 2013;36(4):686-90.

15. Ishiwata $Y$, Itoga $Y$, Yasuhara $M$. Effect of levofloxacin on serum glucose concentration in rats. European J Pharmacol. 2006;551:168-74.

16. Acharya A, Halemani SS, Adake P. Evaluation of Levofloxacin vs. Norfloxacin; Effect on Blood Glucose Profile in Alloxan Induced Diabetic Albino Rats- A Comparative Study. Int J Pharm Sci Res. 2016;7(9):3893-98.

17. Hori S, Kizu J, Kawamura M. Effect of fluoroquinolones on plasma glucose levels in fasted and glucose-loaded mice. $\mathrm{J}$ Infect Chemother. 2006;12:109-111.

18. Maeda N, Tamagawa T, Niki I. Increase in insulin release from rat pancreatic islets by quinolone antibiotics. Br J Pharmacol. 1996;117:372-6.
19. Hamaid NEA, Mostafa IM. Relationship between Ciprofloxacin and blood glucose level, glucose transporters in adult male rats. J Am Sci. 2015;11(3):132-7.

20. Kabbara WK, Ramadan WH, Rahbany P, Natour AS. Evaluation of the appropriate use of commonly prescribed fluoroquinolones and the risk of dysglycemia. Ther Clin Risk Manag. 2015;11:639-47.

21. Murad M, Yglesias CF, Wang A, Sheidaee N, Mullan R, Elamin M, et al. Drug-induced hypoglycemia: a systematic review. J Clin Endocrinol Metab. 2009;3(94):741-5.

Cite this article as: Gupta T, Mulkalwar S, Kulkarni V, Jadhav S, Tilak A, Rane B. A comparative study of effect of fluoroquinolones on blood glucose levels in rats. Int J Basic Clin Pharmacol 2020;9:1217-21. 